\title{
DISORDERED ISING SYSTEMS AND RANDOM CLUSTER REPRESENTATIONS
}

\author{
CHARLES M. NEWMAN* \\ Courant Institute of Mathematical Sciences \\ New York University \\ 251 Mercer Street \\ New York, NY 10012 \\ U.S.A.
}

\begin{abstract}
We discuss the Fortuin-Kasteleyn (FK) random cluster representation for Ising models with no external field and with pair interactions which need not be ferromagnetic. In the ferromagnetic case, the close connections between FK percolation and Ising spontaneous magnetization and the availability of comparison inequalities to independent percolation have been applied to certain disordered systems, such as dilute Ising ferromagnets and quantum Ising models in random environments; we review some of these applications. For non-ferromagnetic disordered systems, such as spin glasses, the state of the art is much more primitive. We discuss some of the many open problems for spin glasses and show how the FK representation leads to one small result, that there is uniqueness of the spin glass Gibbs distribution above the critical temperature of the associated ferromagnet.
\end{abstract}

Key words: FK representations, spin glasses, disordered Ising models, percolation.

\section{The FK Random Cluster Representation}

In this section, we will briefly review the relation between Ising models, FortuinKasteleyn (FK) random cluster models and independent percolation. FK models were introduced in Kasteleyn and Fortuin (1969), Fortuin and Kasteleyn (1972); more recent presentations may be found in Aizenman et al. (1988), Grimmett (1994). Our emphasis here will be on the version relevant for Ising systems with some ferromagnetic and some antiferromagnetic pair interactions; for more discussion of this sort, see Newman (1991). For simplicity, we will restrict attention to models in $\mathbb{Z}^{d}$ with nearest neighbor interactions. Since we will eventually apply the FK representation to disordered systems, we must allow our couplings to vary from bond to bond, in magnitude and in sign.

Let $\widehat{\mathbb{Z}}^{d}$ denote the set of nearest neighbor bonds of $\mathbb{Z}^{d}$; i.e., $\widehat{\mathbb{Z}}^{d}$ is the set of unordered pairs $b=\langle x, y\rangle=\langle y, x\rangle$ of sites $x, y$ in $\mathbb{Z}^{d}$ with Euclidean distance $\| x-$ $y \|=1$. The interactions, $J_{b}$, are real numbers indexed by $b$ in $\widehat{\mathbb{Z}}^{d}$ and the inverse temperature is a non-negative constant $\beta$. (When we consider disordered systems, the $J_{b}$ 's will be random variables on some probability space $(\Omega, \mathcal{F}, P)$ and the present considerations will be relevant for each fixed $\omega \in \Omega$.) Given the $J_{b}$ 's and $\beta$, we define

\footnotetext{
* Supported in part by the National Science Foundation under Grant DMS 92-09053; thanks are due the Isaac Newton Institute for Mathematical Sciences for support and hospitality; NATO for its travel support to attend this Advanced Study Institute; and C. Borgs and J. Bricmont for help with references.
} 
parameters $p_{b} \in[0,1)$ by the formula,

$$
p_{b}=1-e^{-\beta\left|J_{b}\right|} .
$$

For $\Lambda$ a finite subset of $\mathbb{Z}^{d}$, the (volume $\Lambda$ ) Gibbs distribution (with free boundary conditions) for the Ising model is a probability measure on $\{-1,+1\}^{\Lambda}$ and the corresponding FK model distribution is a probability measure on $\{0,1\}^{\Lambda}$, where $\widehat{\Lambda}$ denotes the set of bonds $b=\langle x, y\rangle$ with $x$ and $y$ in $\Lambda$. We regard these respectively as the probability distributions $\mu_{s}$ of +1 or -1 valued spin random variables $\left(S_{x}: x \in \Lambda\right)$ and $\mu_{n}$ of 0 or 1 valued bond occupation variables $\left(N_{b}: b \in \widehat{\Lambda}\right)$. These two measures are the marginal distributions (for their respective sets of variables) of a joint distribution $\mu$ on $\widetilde{\Omega}=\{-1,+1\}^{\Lambda} \times\{0,1\}^{\widehat{\Lambda}}$ defined, in two steps, as follows.

STEP 1. Let $\mu^{\prime}$ be the joint distribution on $\{-1,+1\}^{\Lambda} \times\{0,1\}^{\widehat{\Lambda}}$ of random variables $\left(S_{x}^{\prime}, N_{b}^{\prime}: x \in \Lambda, b \in \widehat{\Lambda}\right)$ which are all mutually independent with $P\left(S_{x}^{\prime}=+1\right)=$ $P\left(S_{x}^{\prime}=-1\right)=\frac{1}{2}$ and $P\left(N_{b}^{\prime}=1\right)=p_{b}$.

STEP 2. Let $\widetilde{U}$ be the event

$$
\widetilde{U}=\left\{\text { for all } b=\langle x, y\rangle \in \widehat{\Lambda}, J_{b} N_{b}^{\prime} S_{x}^{\prime} S_{y}^{\prime} \geq 0\right\}
$$

(regarded as a subset of $\widetilde{\Omega}$ ), and define $\mu$ to be $\mu^{\prime}$ conditioned on $\widetilde{U}$; i.e.,

$$
\mu(\cdot)=\mu^{\prime}(\widetilde{U})^{-1} \mu^{\prime}(\cdot) 1_{\widetilde{U}}(\cdot) .
$$

It is an elementary exercise to show that the two marginal distributions are given explicitly by

$$
\begin{aligned}
& \mu_{s}\left(\left(s_{x}\right)\right)=Z_{s}^{-1} \exp \left(\frac{\beta}{2} \sum_{\langle x, y\rangle \in \widehat{\Lambda}} J_{\langle x, y\rangle} s_{x} s_{y}\right), \\
& \mu_{n}\left(\left(n_{b}\right)\right)=Z_{n}^{-1} 2^{\sharp\left(\left(n_{b}\right)\right)} \mu_{n}^{\text {ind }}\left(\left(n_{b}\right)\right) 1_{U}\left(\left(n_{b}\right)\right),
\end{aligned}
$$

where $Z_{s}$ and $Z_{n}$ are normalization constants, $\sharp\left(\left(n_{b}\right)\right)$ denotes the number of clusters determined by $\left(n_{b}\right)$ (i.e., the number of connected components in the graph with vertex set $\Lambda$ and edge set, $\left.\left\{b \in \widehat{\Lambda}: n_{b}=1\right\}\right), \mu_{n}^{\text {ind }}$ is the Bernoulli product measure corresponding to independent occupation variables with $\mu_{n}^{\text {ind }}\left(\left\{n_{b}=1\right\}\right)=p_{b}$ for each $b$ and $U$ is the event in $\{0,1\}^{\widehat{\Lambda}}$,

$$
\begin{aligned}
U=\left\{\left(n_{b}\right):\right. & \text { there exists some choice of }\left(s_{x}: x \in \Lambda\right) \\
& \text { so that } \left.\left(\left(s_{x}\right),\left(n_{b}\right)\right) \in \widetilde{U}\right\} .
\end{aligned}
$$

The formula (1.4) is standard for an Ising model Gibbs distribution. Likewise (1.5) is standard for the FK model in the ferromagnetic case $\left(J_{b} \geq 0\right.$ for all $\left.b\right)$, since then $U=\{0,1\}^{\widehat{\Lambda}}$ (by taking $s_{x} \equiv+1$ or $\equiv-1$ in (1.6)). FK models for nonferromagnetic interactions are less well known; the first published reference we are aware of is Kasai and Okiji (1988) (see also Swendsen and Wang 1987, Edwards and Sokal 1988, Newman 1991). Here $U$, which is typically not all of $\{0,1\}^{\widehat{\Lambda}}$, may be 
thought of as the set of 'unfrustrated' bond occupation configurations. This term, borrowed from the spin glass literature, simply means that for the Ising Hamiltonian restricted to occupied bonds,

$$
H_{\left(n_{b}\right)}\left(\left(s_{x}\right)\right)=\frac{1}{2} \sum_{b=\langle x, y\rangle \in \widetilde{\Lambda}}\left(-J_{b} n_{b} s_{x} s_{y}\right),
$$

there is some spin configuration $\left(s_{x}\right)$ which simultaneously minimizes each summand.

A key feature of the measure $\mu$, given by (1.3), is that the conditional distribution, $\mu\left(\left(s_{x}\right) \mid\left(n_{b}\right)\right)$, for the $S_{x}$ 's given the $N_{b}$ 's is particularly simple: consider the clusters determined by the given $\left(n_{b}\right)$. Any two sites $u, v$ in the same cluster (which we write as $u \leftrightarrow v$ ) are connected by a path of occupied bonds (with non-zero interactions on every edge) which, because of the conditioning on $\widetilde{U}$ in (1.3), requires that $S_{u}=$ $\eta_{u, v} S_{v}$ where $\eta_{u, v}\left(\left(n_{b}\right)\right)$ is the product of the signs of the $J_{b}$ 's along the occupied path between $u$ and $v$. Two different paths will give the same $\eta$ providing $\left(n_{b}\right) \in U$. For future use, we extend the definition of $\eta_{u, v}\left(\left(n_{b}\right)\right)$ to be 0 if $u$ and $v$ are not in the same cluster for the given $\left(n_{b}\right)$. Thus the relative signs of all the spin variables in a single $\left(n_{b}\right)$-cluster are determined by $\left(n_{b}\right)$ but the spin of any single variable may be either +1 or -1 . The conditional distribution $\mu\left(\left(s_{x}\right) \mid\left(n_{b}\right)\right)$ corresponds to making the \pm 1 choices for each $\left(n_{b}\right)$-cluster by independent flips of a fair coin. (The conditional distribution $\mu\left(\left(n_{b}\right) \mid\left(s_{x}\right)\right)$ is also very simple (Swendsen and Wang 1987), but we will not make use of that.)

Expressing $\mu$ as the product of the marginal $\mu_{n}$ and the above conditional allows one to express $\mu_{s}$ expectations (which we write $E_{s}$ ) in terms of $\mu_{n}$ expectations (which we write $E_{n}$ ). This is the sense in which the FK model gives a representation of the Ising model. For example,

$$
E_{s}\left(S_{u} S_{v}\right)=E_{n}\left(\eta_{u, v}\right)
$$

which, in the ferromagnetic case (where $\eta_{u, v}$ can only be +1 or 0 ) becomes the well known formula

$$
E_{s}\left(S_{u} S_{v}\right)=\mu_{n}(u \leftrightarrow v) .
$$

To continue our presentation, we now introduce boundary conditions. The simplest type of boundary condition is an assignment $\bar{s}=\left(\bar{s}_{z}\right)$ of \pm 1 spin values to the sites $z$ in $\partial \Lambda$, the set of sites outside of $\Lambda$ which are nearest neighbors of sites in $\Lambda$. Here it is convenient to replace $\Lambda$ by $\Lambda^{*}=\Lambda \cup \partial \Lambda$ and $\widehat{\Lambda}$ by $\widehat{\Lambda}^{*}$, the union of $\widehat{\Lambda}$ and bonds $\langle x, y\rangle$ with $x \in \Lambda$ and $y \in \partial \Lambda$; i.e., $\mu$ will be replaced by a measure $\mu^{\bar{s}}$ on $\Omega^{*}=\{-1,+1\}^{\Lambda^{*}} \times\{0,1\}^{\Lambda^{*}}$. The definition of $\mu^{\bar{s}}$ is just like that of $\mu$, except that in Step $1, S_{x}^{\prime}$ is set to $\bar{s}_{x}$ for each $x \in \partial \Lambda$. In the formulas for the marginal distributions, (1.4) is replaced by the usual Ising model Gibbs distribution formula with boundary condition $\bar{s}$, while (1.5) remains essentially the same. We note however that in the definition of $U$ (and $\widetilde{U}$ ) the spins in $\partial \Lambda$ are always fixed by $\bar{s}$, and further that $\sharp\left(\left(n_{b}\right)\right)$ only counts clusters which do not touch $\partial \Lambda$ (or equivalently for the definition of $\mu_{n}$, counts all clusters touching the boundary as a single cluster).

Note that even in the ferromagnetic case, $U$ is generally not all of $\{0,1\} \widehat{\Lambda}^{*}$ since occupied paths of $J_{b}>0$ bonds are not allowed to connect the $\bar{s}_{z}=+1$ and $\bar{s}_{z}=-1$ 
parts of the boundary. Of course $U$ will be all of $\{0,1\}^{\Lambda^{*}}$ in the ferromagnetic case if $\bar{s}_{z} \equiv+1$ or $\bar{s}_{z} \equiv-1$; the resulting marginal distributions are denoted $\mu_{s}^{+}, \mu_{s}^{-}$and (for either +1 or -1$) \mu_{n}^{w}$ ( $w$ for 'wired').

The conditional distribution $\mu\left(\left(s_{x}\right) \mid\left(n_{b}\right)\right)$ remains as it was in the free boundary condition case except that no coin is tossed for clusters touching the boundary since their spin values are already determined by $\left(n_{b}\right)$ and $\bar{s}$ (and the signs of the $J_{b}$ 's). In the ferromagnetic case, the (finite volume) magnetization at site $u$ (in $\Lambda$ ) is then

$$
E_{s}^{+}\left(S_{u}\right)=\mu_{n}^{w}(u \leftrightarrow \partial \Lambda)=-E_{s}^{-}\left(S_{u}\right),
$$

where of course $\mu \leftrightarrow \partial \Lambda$ means that the $\left(n_{b}\right)$-cluster containing the site $u$ touches the boundary.

Here are some easily derived comparison inequalities. For a given $\Lambda$, write $\mu_{n,\left(p_{b}\right)}^{\text {ind }}$ and $\mu_{n,\left(p_{b}\right)}^{w, F}$ to denote the two probability measures on $\{0,1\}^{\widehat{\Lambda}^{*}}$ given respectively as the Bernoulli product measure with parameters $\left(p_{b}\right)$, and as the wired b.c. ferromagnetic $\left(J_{b} \geq 0\right)$ FK-measure with the same parameters. Write $\mu_{1} \ll \mu_{2}$ to denote stochastic ordering between measures; i.e., to denote that $\int f d \mu_{1} \leq \int f d \mu_{2}$ for any coordinate-wise increasing real function $f$ on $\{0,1\}^{\widehat{\Lambda}^{*}}$. Then

$$
\mu_{n,\left(h\left(p_{b}\right)\right)}^{\text {ind }} \ll \mu_{n,\left(p_{b}\right)}^{w, F} \ll \mu_{n,\left(p_{b}\right)}^{\text {ind }},
$$

where

$$
h\left(p_{b}\right)=\frac{p_{b}}{2\left(1-p_{b}\right)+p_{b}} .
$$

These inequalities can be derived using only the fact (Harris 1960) that for the independent percolation measure $\mu_{n}^{\text {ind }}$, increasing functions $f$ and $g$ positively correlated. Using the facts that these FKG inequalities are also valid for $\mu_{n}^{w, F}$ and further that the density of (a non-ferromagnetic) $\mu_{n,\left(p_{b}\right)}^{\bar{s}}$ with respect to $\mu_{n,\left(p_{b}\right)}^{w, F}$ is, according to (1.5), proportional to the decreasing function $1_{U}$, it follows that

$$
\mu_{n,\left(\beta J_{b}\right)}^{\bar{s}} \ll \mu_{n,\left(p_{b}\right)}^{w, F} ;
$$

here we use $\left(\beta J_{b}\right)$ as a subscript on the left-hand side because of the dependence on the signs of the $\beta J_{b}$ 's (and not just on their magnitudes through the $p_{b}$ 's). We note that the obvious analogue of (1.13) is valid when both sides have free boundary conditions; analogues involving more general boundary conditions will be discussed in Section 3 below.

One consequence of inequalities such as (1.13) is that the spin correlations of a non-ferromagnetic Ising model are dominated by those of the associated ferromagnet. This domination was already noted (in a homework problem) by Griffiths (1971). In Section 3 we derive some other consequences. 


\section{The Phase Transition for Dilute Ferromagnets}

Throughout this section we restrict attention to ferromagnetic Ising and FK models. For fixed $\left(J_{b}: b \in \widehat{\mathbb{Z}}^{d}\right)$, we denote in this section by $\mu_{s}^{+}$and $\mu_{n}^{w}$ the infinite volume limits $\left(\Lambda \rightarrow \mathbb{Z}^{d}\right)$ of the corresponding finite $\Lambda$ measures defined in the last section; these limits are known to exist by various monotonicity in $\Lambda$ arguments, based on the ferromagnetic nature of the interactions. The infinite volume limit of (1.10) is

$$
E_{s}^{+}\left(S_{u}\right)=\mu_{n}^{w}(u \leftrightarrow \infty),
$$

where $u \leftrightarrow \infty$ denotes the event that the $\left(n_{b}\right)$-cluster containing site $u$ is infinite. Other arguments (Lebowitz and Martin-Löf 1972) based on FKG inequalities, show that ferromagnetic Ising models have a unique infinite volume Gibbs distribution if and only if $E_{s}^{+}\left(S_{u}\right)=0$ for all $u$ and thus by (2.1) if and only if

$$
\mu_{n}^{w}(\text { some cluster is infinite })=0 .
$$

Thus, for ferromagnetic systems, a phase transition for the Ising model (in the sense of a transition from unique to multiple infinite volume Gibbs distributions) is precisely equivalent to a percolation phase transition for the corresponding wired b.c. $F K$ measure. We denote by $\beta_{c}=\beta_{c}\left(\left(J_{b}: b \in \mathbb{Z}^{d}\right)\right)$ the critical inverse temperature for this phase transition.

For the remainder of this section, we follow the analysis of Aizenman et al. (1987) which shows how this fact may be combined with the comparison inequalities (1.11) to yield an elegant analysis of disordered, but still ferromagnetic, Ising models. In these models, the interactions $\left(J_{b}: b \in \widehat{\mathbb{Z}}^{d}\right)$ will be non-negative i.i.d. random variables on some probability space $(\Omega, \mathcal{F}, P)$. The critical inverse temperature $\beta_{c}$ does not depend on any finite number of the $J_{b}$ 's and hence, by the Kolmogorov zero-one law is a.s. a constant.

Let us denote the density of active bonds by $p^{\prime}=P\left(J_{b} \neq 0\right)$ (which we assume is strictly positive) and denote the critical value for standard nearest neighbor independent bond percolation on $\mathbb{Z}^{d}$ by $p_{c}$. The percolation probability (for the independent model) is

$$
\theta(p)=\mu_{n,(p)}^{\text {ind }}(u \leftrightarrow \infty),
$$

where $\mu_{n,(p)}^{\text {ind }}$ denotes the Bernoulli product measure on $\{0,1\}^{\widehat{Z}^{d}}$ corresponding to the independent percolation model with occupation density $p$. We recall that by the definition of $p_{c}, \theta(p)=0$ for $p<p_{c}$ and $\theta(p)>0$ for $p>p_{c}$, but there is still no proof that $\theta\left(p_{c}\right)=0$ for all $d(\geq 2)$.

There are two facts about the dependence of $\beta_{c}$ on the distribution of $J_{b}$ (including its dependence on $p^{\prime}$ ) which are easily derived without use of the FK representation: first, that if ( $d \geq 2$ and) $P\left(J_{b}<\epsilon\right)=0$ for some $\epsilon>0$, then $\beta_{c}<\infty$, and second that if $\theta\left(p^{\prime}\right)=0$ then $\beta_{c}=\infty$ (i.e., there is (a.s.) a unique infinite volume Ising model Gibbs distribution for any $\beta<\infty$ ). The next theorem, based on the FK representation, improves these results considerably. It was used in Aizenman et al. (1987) primarily to analyze the rate of divergence of $\beta_{c}\left(p^{\prime}\right)$ as $p^{\prime} \downarrow p_{c}$ in the classic dilute ferromagnet, where $J_{b}$ takes on only the values 0 and 1 . 
Theorem 1. (Aizenman et al. 1987) For a given distribution of $J_{b}$ and value of $\beta$, define two constants:

$$
\bar{p}=E\left(1-e^{-\beta J_{b}}\right), \quad \underline{p}=E\left(\frac{1-e^{-\beta J_{b}}}{2 e^{-\beta J_{b}}+1-e^{-\beta J_{b}}}\right) .
$$

The infinite volume Ising model Gibbs distribution is (a.s.) unique if $\bar{p}<p_{c}$ and is (a.s.) non-unique if $\underline{p}>p_{c}$. Thus $\beta_{c}<\infty$ if and only if $p^{\prime} \equiv P\left(J_{b} \neq 0\right)>p_{c}$.

Proof. According to the FK-percolation criterion for the Ising phase transition, we need to show that the non-percolation property (2.2) for $\mu_{n}^{w}=\mu_{n,\left(p_{b}\right)}^{w}$ is (a.s.) valid when $\bar{p}<p_{c}$ and (a.s.) invalid when $\underline{p}>p_{c}$. (We then leave the proof of the last statement of the theorem to the reader.) Let us consider the probability measure

$$
\widetilde{\mu}_{n}=E\left(\mu_{n,\left(p_{b}\right)}^{w}\right),
$$

where as usual $E$ denotes expectation with respect to the probability measure $P$ for the $J_{b}$ 's; $\widetilde{\mu}_{n}$ represents the marginal distribution of the FK bond occupation variables when the $J_{b}$ 's are not conditioned on. It suffices to show that

$$
\widetilde{\mu}_{n}(\text { some cluster is infinite })=\left\{\begin{array}{lll}
0, & \text { if } & \bar{p}<p_{c} \\
1, & \text { if } & \underline{p}>p_{c}
\end{array}\right.
$$

since this implies the corresponding identity for $P$-a.e. $\mu_{n,\left(p_{b}\right)}^{w}$. Now we use (the infinite volume limit of) the comparison inequalities (1.11) and average them over the $\left(p_{b}\right)$ 's to obtain

$$
\mu_{n, \underline{p})}^{\mathrm{ind}}=E \mu_{n,\left(h\left(p_{b}\right)\right)}^{\mathrm{ind}} \ll \widetilde{\mu}_{n} \ll E \mu_{n,\left(p_{b}\right)}^{\mathrm{ind}}=\mu_{n,(\bar{p})}^{\mathrm{ind}},
$$

from which the proof is easily completed. The equalities of (2.7) are basically trivial; e.g., in the $J_{b}=0$ or 1 case, they may be restated as follows. If bonds are independently declared active with probability $p^{\prime}$ and then active bonds are independently declared occupied with probability $p$, the resulting occupied bonds form an independent percolation model with occupation probability $p p^{\prime}$.

To complete this section we briefly mention another type of disordered ferromagnet where an FK representation has been used (Aizenman et al. 1993). This is the quantum Ising model on $\mathbb{Z}^{d}$ with random couplings and a random transverse field. A Feynman-Kac type approach (see Aizenman and Nachtergaele 1993) represents this quantum model in terms of a classical Ising model, where the Ising spin variables are indexed by $\mathbb{Z}^{d} \times \mathbb{R}$. The disorder in this representation remains $d$-dimensional, so that nearest neighbor couplings, which depend (randomly) on the location in $\mathbb{Z}^{d}$, do not depend on the $\mathbb{R}$-coordinate (the 'time'). There is an FK representation for this classical Ising model which is related to independent percolation models on $\mathbb{Z}^{d} \times \mathbb{R}$ in essentially the same way as in the discrete index setting. These percolation models are related to the graphical representation of contact processes in the same way as ordinary percolation is related to oriented percolation; in particular the $d$-dimensional disorder corresponds exactly to the random environment natural for a contact process on $\mathbb{Z}^{d}$. We note that by using the natural string of identities 
and comparison inequalities provided by the FK representation, one new result for the original disordered quantum model is shown in Aizenman et al. (1993) to follow from a known result of Liggett (1992) about the contact process in a random environment. [Warning: In Section 4 of Aizenman et al. (1993), the definition of $B$ being an encounter region should be modified to include the requirement that the event $G_{B}$ occurs; without this change, the combinatorial part of the proof of Prop. 4.1 there (uniqueness of the infinite cluster) would be incorrect.]

\section{Spin Glasses: Results on Uniqueness}

The spin glass models we will consider (Edwards and Anderson 1975) are Ising models with nearest neighbor interactions $\left(J_{b}: b \in \widehat{\mathbb{Z}}^{d}\right)$ which are i.i.d. symmetric random variables on some $(\Omega, \mathcal{F}, P)$, as likely to be negative as positive. A good special case to keep in mind is where $J_{b}=+1$ or -1 , each with probability $\frac{1}{2}$. A standard review article on spin glasses is Binder and Young (1986). In the next section we will discuss the open problem of proving that for $d$ and $\beta$ sufficiently large, there is (a.s.) non-uniqueness of the Gibbs distribution for such models; here we consider the converse issue.

It seems generally accepted in the physics literature (see Binder and Young 1986) that, for $d=2$, there should be (a.s.) a unique Gibbs distribution for all $\beta<\infty$ (at least for reasonable distributions of the $J_{b}$ 's such as Gaussian or the \pm 1 valued case). It is an interesting open problem to prove this conjecture; it should be noted that an analogous result was proved for the $d=2$ random field Ising model in Aizenman and Wehr (1990). Since we are unable to resolve the $d=2$ large $\beta$ problem, we will instead show how FK methods lead to some small progress on the rather less interesting issue of general $d$ and moderate values of $\beta$.

First we note that in any dimension, uniqueness for sufficiently large $\beta$ can be proved (under some restrictions on the distribution of the $J_{b}$ 's) by DobrushinShlosman techniques, which are insensitive to the signs of the interactions (see Dobrushin 1968 and Dobrushin and Shlosman 1985). Let us denote by $\beta_{c}^{F}$, the critical temperature for the associated disordered ferromagnet in which each spin glass interaction $J_{b}$ is replaced by $\left|J_{b}\right|$. (For the case of \pm 1 valued $J_{b}$ 's, this ferromagnet will of course not be disordered.) It seems intuitively clear that uniqueness for the ferromagnet should imply uniqueness for the spin glass (in particular, for $\beta<\beta_{c}^{F}$ ), but this does not seem to follow from the above mentioned techniques. We will now show that such a result can be derived by FK techniques. The result in fact has nothing to do with disordered systems at all:

Theorem 2. For a given set of real valued interactions $\left\{J_{b}: b \in \widehat{\mathbb{Z}}^{d}\right\}$, uniqueness of the infinite volume Gibbs distribution at inverse temperature $\beta$ for the associated ferromagnetic interactions, $\left\{\left|J_{b}\right|: b \in \widehat{\mathbb{Z}}^{d}\right\}$, implies uniqueness at the same $\beta$ for the original interactions.

Proof. The proof uses the FK representation, the comparison inequality (1.13) and a coupling argument (based on a generalization of (1.13)). All but the coupling have been discussed previously (see Newman 1991) and that argument is similar to one used recently by van den Berg and Maes (1992). Let $S_{A}=\prod_{u \in A} S_{u}$ for any fixed 
finite $A \subset \mathbb{Z}^{d}$ and let $\Lambda$ be a (varying) finite subset of $\mathbb{Z}^{d}$ containing $A$; it suffices to show that for the original set of interactions, any two choices of boundary conditions $\bar{s}=\bar{s}(\Lambda)$ and $\bar{s}^{\prime}=\bar{s}^{\prime}(\Lambda)$ have

$$
E_{s, \Lambda}^{\bar{s}}\left(S_{A}\right)-E_{s, \Lambda}^{\bar{s}^{\prime}}\left(S_{A}\right) \rightarrow 0 \quad \text { as } \quad \Lambda \rightarrow \mathbb{Z}^{d} .
$$

Here we have added a subscript to indicate dependence on $\Lambda$. The idea is to express each of the two expectations as (asymptotically) the same mixture (over regions $\widetilde{\Lambda}$ ) of free boundary condition expectations.

We begin by noting that it easily follows from the FK representation that for a given $\widetilde{\Lambda} \subset \Lambda$, conditioned on the bond occupation variables other than those entirely in $\widetilde{\Lambda}$, if $n_{b}=0$ for every $b$ between $\widetilde{\Lambda}$ and $\partial \widetilde{\Lambda}$, then the conditional distribution for the bonds and spins in $\widetilde{\Lambda}$ is just the volume $\widetilde{\Lambda}$ measure with free boundary conditions.

Now for the $\Lambda$ in question, we wish to couple the measures $\mu_{n, \Lambda}^{\bar{s}}$ for the original interactions with b.c. $\bar{s}$ and $\mu_{n, \Lambda}^{w, F}$ for the ferromagnetic interactions $\left|J_{b}\right|$ and wired boundary condition; i.e., realize the corresponding variables $N_{b}^{\bar{s}}$ and $N_{b}^{w, F}$ on the same probability space $\left(\Omega^{\prime}, \mathcal{F}^{\prime}, P^{\prime}\right)$. Let us denote by $C_{\Lambda}^{w}$ the $\mathrm{N}_{b}^{w, F}$-cluster of $\partial \Lambda$, i.e., the set of sites in $\Lambda$ which are connected to $\partial \Lambda$ by a path of bonds with $N_{b}^{w, F}=1$. We need our coupling to have two properties. First, that if $\Lambda \backslash C_{\Lambda}^{w}=\widetilde{\Lambda} \neq \emptyset$, then $N_{b}^{\bar{s}}=0$ for every $b$ between $\widetilde{\Lambda}$ and $\partial \widetilde{\Lambda}$; this would follow from the pointwise domination $N_{b}^{\bar{s}}\left(\omega^{\prime}\right) \leq N_{b}^{w, F}\left(\omega^{\prime}\right)$. Second, that conditional on $\Lambda \backslash C_{\Lambda}^{w}=\widetilde{\Lambda} \neq \emptyset$, the conditional distribution of $\left(N_{b}^{\bar{s}}: b=\langle x, y\rangle\right.$ with $\left.x, y \in \widetilde{\Lambda}\right)$ is the free boundary condition FK measure on $\widetilde{\Lambda}$. It is a standard fact that the first property follows from (1.13); we claim that both properties can be had simultaneously by a sequential construction (i.e., one bond $b$ at a time) using (1.13) and a family of analogous inequalities involving more general boundary conditions, which we now discuss. Further details needed to justify our claim are left to the reader; we note that some care should be taken in choosing the (random) order of bonds in the construction so that the second property needed for the coupling will be valid. We also note that the coupling construction is quite similar to the one used by van den Berg and Maes (1992).

First we note that two fixed spin boundary conditions related by an overall spin flip, $\bar{s}$ and $-\bar{s}$, give rise to exactly the same FK measure. Thus the boundary conditions appearing in (1.13) are really defined by an assignment of relative signs to the sites on the boundary. The generalized boundary conditions we consider are as follows. Let $\Lambda$ be a finite subset of $\mathbb{Z}^{d}$ and let $\widehat{\Lambda}^{\prime}$ be a non-empty subset of $\widehat{\Lambda}$, the set of nearest neighbor bonds between sites in $\Lambda$. A general boundary condition $\theta$ for the FK measure $\mu_{n,\left(\beta J_{b}\right)}^{\theta}$ on $\{0,1\}^{\widehat{\Lambda}^{\prime}}$ is specified by a partition of $\Lambda$ into non-empty subsets $\Lambda_{1}, \Lambda_{2}, \ldots, \Lambda_{m}$ and an assignment $t_{i}$ of relative signs to the sites within each $\Lambda_{i}$ with at least two sites. The formula for this measure is given by (1.5), where $\sharp\left(\left(n_{b}\right)\right)$ treats all sites in $\Lambda_{i}$ as already being in the same cluster, for each $i$, and where the definition (1.6) for $U$ is modified to allow only $\left(s_{x}: x \in \Lambda\right)$ which respect all the $t_{i}$ 's. The free boundary condition case corresponds to the partition of $\Lambda$ entirely into individual sites (so no $t_{i}$ 's are assigned) while the $\bar{s}$ boundary condition on the boundary $\partial \Gamma$ of some region $\Gamma$ corresponds to taking $\Lambda=\Gamma^{*}(=\Gamma \cup \partial \Gamma), \widehat{\Lambda}^{\prime}=\widehat{\Gamma}^{*}$ (see the definition following (1.9) above), $\Lambda_{1}=\partial \Gamma$, all other $\Lambda_{i}$ 's as individual sites of $\Gamma$ and finally $t_{1}$ as the relative sign assignment given by $\bar{s}$. 
The analogues of (1.13) are as follows, for a given $\Lambda$ and $\widehat{\Lambda}^{\prime}$. Let $\kappa^{\prime}$ be a boundary condition in which each $t_{i}^{\prime}$ is the assignment that all sites in $\Lambda_{i}^{\prime}$ have the same sign. The ferromagnetic FK measure with such a 'partially wired' boundary condition, $\mu_{n,\left(p_{b}\right)}^{\kappa^{\prime}, F}$, still obeys the FKG inequalities. Now let $\kappa$ be another boundary condition whose partition of $\Lambda$ into $\Lambda_{1}, \Lambda_{2}, \ldots$ is a refinement of the $\kappa^{\prime}$ partition into $\Lambda_{1}^{\prime}, \Lambda_{2}^{\prime}, \ldots$ (i.e., each $\Lambda_{j}^{\prime}$ is a union of some of the $\Lambda_{i}$ 's). Then, with no restriction on the assignments $t_{1}, t_{2}, \ldots$ for $\kappa$, we claim that

$$
\mu_{n,\left(\beta J_{b}\right)}^{\kappa} \ll \mu_{n,\left(p_{b}\right)}^{\kappa^{\prime}, F} .
$$

To verify this domination, note that the density of the left-hand side with respect to the right-hand side is proportional to $1_{U} \cdot 2^{\sharp-\sharp^{\prime}}$, which is a decreasing function because both $1_{U}$ and $\sharp-\sharp^{\prime}$ are decreasing. The domination then follows from the FKG inequalities for the right-hand side.

By using our coupled bond measure and then constructing Ising spins by the usual coin tossing procedure for the $N_{b}^{\bar{s}}$-clusters, we may express the b.c. $\bar{s}$ expectation as mostly a mixture of free b.c. expectations:

$$
E_{s, \Lambda}^{\bar{s}}\left(S_{A}\right)=\sum_{\Lambda \supseteq \widetilde{\Lambda} \supset A} \mu_{n, \Lambda}^{w, F}\left(\Lambda \backslash C^{w}=\widetilde{\Lambda}\right) \cdot E_{s, \widetilde{\Lambda}}\left(S_{A}\right)+\mu_{n, \Lambda}^{w, F}(A \leftrightarrow \partial \Lambda) \theta,
$$

where $\theta$ is the conditional expectation of $S_{A}$ given that the $N_{b}^{w, F}=1$ bonds connect $A$ to $\partial \Lambda$. A similar formula will be valid for the other b.c. $\bar{s}^{\prime}$ with the same righthand side except that $\theta$ will be replaced by $\theta^{\prime}$. Since $|\theta|,\left|\theta^{\prime}\right| \leq 1$ (because $S_{A}= \pm 1$ ), (3.1) will follow from

$$
\mu_{n, \Lambda}^{w, F}(A \leftrightarrow \partial A) \rightarrow 0 \quad \text { as } \quad \Lambda \rightarrow \mathbb{Z}^{d},
$$

for fixed $A$. But this is equivalent to the lack of percolation in the infinite volume limit of $\mu_{n, \Lambda}^{w, F}$ which is equivalent to uniqueness of the ferromagnetic infinite volume Gibbs distribution, as explained at the beginning of Section 2.

REMARKs. The proof of Theorem 2 immediately yields the following specific bound on the influence of boundary conditions:

$$
\text { for } A \subset \Lambda,\left|E_{s, \Lambda}^{\bar{s}}\left(S_{A}\right)-E_{s, \Lambda}^{\bar{s}^{\prime}}\left(S_{A}\right)\right| \leq 2 \sum_{x \in A} E_{s, \Lambda}^{+, F}\left(S_{x}\right) \text {. }
$$

Essentially the same arguments show that at any $\beta$ where there is a unique infinite volume Gibbs distribtuion $\mu_{s}^{F}$ for $\left(\beta\left|J_{b}\right|\right)$, the correlation decay properties of the unique Gibbs distribution $\mu_{s}$ for $\left(\beta J_{b}\right)$ are controlled by:

$$
\left|E_{s}\left(S_{A} S_{B}\right)-E_{s}\left(S_{A}\right) E_{s}\left(S_{B}\right)\right| \leq \sum_{\substack{x \in A \\ y \in B}} E_{s}^{F}\left(S_{x} S_{y}\right) .
$$

Finally, we remark that for i.i.d. $J_{b}$ 's with an arbitrary (not necessarily symmetric) common distribution, an explicit high temperature regime with a.s. uniqueness and exponential decay of correlations can be easily obtained by combining (i) (3.5)-(3.6), (ii) the Aizenman et al. (1987) analysis of random ferromagnets (see Theorem 1 and especially (2.7) above), (iii) known results about standard (subcritical) independent percolation and (iv) some elementary arguments. 


\section{Spin Glasses: Non-Results on Non-Uniqueness}

As mentioned previously, it is an open problem to prove that for spin glasses in sufficiently high dimensions, there are (a.s.) multiple infinite volume Gibbs distributions for large $\beta$. (See Reger et al. 1990 for numerical results with $d=4$.) The proof of Theorem 2 suggests (but does not prove) that a necessary condition for non-uniqueness of Gibbs distributions is the occurrence of percolation in infinite volume FK measures. We note that for non-ferromagnetic models, there is neither a specially distinguished boundary condition (such as wired in the ferromagnetic case) nor a guarantee of a single infinite volume limit (as $\Lambda \rightarrow \mathbb{Z}^{d}$ ) for any particular choice of boundary conditions. Thus, to consider percolation or any other property of infinite volume FK measures, we must worry about what procedure we use to specify an infinite volume FK measure for each (or almost each) $\omega$ in the probability space for the $J_{b}$ 's. This same issue will arise again when we discuss later the various types of non-uniqueness which could occur in spin glasses.

For a finite volume $\Lambda$, we will expand the types of boundary conditions we considered in Section 1 (for spins or bonds or for both together) beyond free and specific $\bar{s}$ to include also a mixture over choices of $\bar{s}$ (for the given $\Lambda$ ); also if $\Lambda$ is a cube (or rectangular parallelipiped) we will allow periodic (or antiperiodic in one or more coordinate directions) boundary conditions. We will often have cause to distinguish between boundary conditions which do or do not depend on $\omega$; of course the boundary condition will in general depend on $\Lambda$. When taking subsequence limits as $\Lambda \rightarrow \mathbb{Z}^{d}$, we will also distinguish between subsequences which do or do not depend on $\omega$.

One way to obtain an infinite volume spin and bond measure for almost every $\omega$ is to choose a (measurably) $\omega$-dependent boundary condition for each $\Lambda$, consider the resulting joint distribution (for a given $\beta$ ) on $\Omega \times\{$ spin and bond configurations $\}$ and take some subsequence limit which will be a measure, denoted by $P^{*}$, on $\Omega \times\{+1,-1\}^{\mathbb{Z}^{d}} \times\{0,1\}^{\widehat{\mathbb{Z}}^{d}}$ (with the natural product $\sigma$-field). (It is best at this stage to assume that $\Omega$ is the canonical product space $\mathbb{R}^{\widehat{\mathbb{Z}}^{d}}$.) By construction, the subsequence of $\Lambda$ 's chosen does not depend on $\omega$ here. Clearly the marginal distribution of $P^{*}$ on $\Omega$ will just be $P$ and it is not difficult to see that for $P$-a.e. $\omega$, the conditional distribution $\mu_{s}^{\omega}$ of the spin variables (given $\omega$ ) will be an infinite volume Gibbs distribution for the interactions $\left(J_{b}(\omega)\right)$. We will focus attention first on the conditional distribution of the bond occupation variables $\mu_{n}^{\omega}$ (given $\omega$ ), which is our infinite volume FK-measure. The next theorem is a slight extension of a result in Gandolfi et al. (1992).

Theorem 3. (Gandolfi et al. 1992) Let $\mu_{n}^{\omega}$ be an infinite volume FK-measure on $\{0,1\}^{\mathbb{Z}^{d}}$, obtained as described above, but with $\omega$-independent boundary conditions. Let $\bar{p}=E\left(1-e^{-\beta\left|J_{b}\right|}\right)$ and let $p_{c}$ be the critical value for standard independent bond percolation on $\mathbb{Z}^{d}$, as in Theorem 1. If $\frac{1}{2} \bar{p}>p_{c}$, then a.s. (i.e., for P-almost every $\omega)$

$$
\mu_{n}^{\omega}(\text { some cluster is infinite })=1 \text {; }
$$

in particular (4.1) will be the case for sufficiently large $\beta$ if $d>2$ and $P\left(J_{b}=0\right)=0$. 
REMARK. As will be clear from the proof, (4.1) is also valid for large $\beta$ when $P\left(J_{b}=0\right)=0$, for spin glass models on any lattice where the critical value for standard bond percolation is strictly below $\frac{1}{2}$; this includes $\mathbb{Z}^{2} \times\{0,1\}$. It is an open problem to extend this result to $\mathbb{Z}^{2}$; this would not contradict the conjecture that there is uniqueness of spin glass Gibbs distributions for $d=2$ for reasons we will discuss below.

Proof. We will show that (4.1) is a consequence of $\frac{1}{2} \bar{p}>p_{c}$; the final statement of the theorem then follows from the facts that $\bar{p} \rightarrow P\left(J_{b} \neq 0\right)$ as $\beta \rightarrow \infty$ and that $p_{c}<\frac{1}{2}$ for $d>2$. As in the proof of Theorem 1, we will prove the a.s. validity of (4.1) by averaging $\mu_{n}^{\omega}$ over $\omega$, yielding the marginal distribution of the bond variables, $\tilde{\mu}_{n}=E\left(\mu_{n}\right)$, and then showing that

$$
\tilde{\mu}_{n} \gg \mu_{n,(\bar{p} / 2)}^{\text {ind }} .
$$

To prove (4.2) for the infinite volume measure $\tilde{\mu}_{n}$, it suffices to prove the analogous inequality for the corresponding measure $\tilde{\mu}_{n}^{\kappa}$ on the finite region $\Lambda$ with the specified ( $\omega$-independent) boundary condition $\kappa . \tilde{\mu}_{n}^{\kappa}$, which is an average over $\omega$ 's of FK measures $\mu_{n,\left(\beta J_{b}\right)}^{\kappa}$ may be obtained by first taking the conditional expectation given all the $\left|J_{b}\right|$ 's (which we denote $\bar{\mu}_{n,\left(p_{b}\right)}^{\kappa}$ ) and then averaging over the $\left|J_{b}\right|$ 's (or $p_{b}$ 's). We claim that (a.s.)

$$
\bar{\mu}_{n,\left(p_{b}\right)}^{\kappa} \gg \mu_{n,\left(p_{b} / 2\right)}^{\text {ind }}
$$

since the average (over $p_{b}$ 's) of the right-hand side of $(4.3)$ is just $\mu_{n,(\bar{p} / 2)}^{\text {ind }}$ (recall the equalities of (2.7)), the desired (4.2) would follow.

By a standard argument, (4.3) would be a consequence of the family of inequalities (for all bonds $b^{*}$, and all $n_{b}=0$ or 1 for $b \neq b^{*}$ )

$$
\frac{\bar{\mu}_{n,\left(p_{b}\right)}^{\kappa}\left(N_{b^{*}}=1, N_{b}=n_{b} \text { for } b \neq b^{*}\right)}{\bar{\mu}_{n,\left(p_{b}\right)}^{\kappa}\left(N_{b^{*}}=0, N_{b}=n_{b} \text { for } b \neq b^{*}\right)} \geq \frac{\frac{1}{2} p}{1-\frac{1}{2} p} .
$$

Since (given the $\left|J_{b}\right|$ 's) the signs of the $J_{b}$ 's are i.i.d. symmetric \pm 1 valued random variables, the left-hand side of (4.4) may be expressed (using (1.5)) as

$$
\frac{p_{b} \sum_{\alpha}\left(C_{\alpha}^{+} 2^{-H_{\alpha}} I_{\alpha}^{+}(1)+C_{\alpha}^{-} 2^{-H_{\alpha}} I_{\alpha}^{-}(1)\right)}{\left(1-p_{b}\right) \sum_{\alpha}\left(C_{\alpha}^{+} I_{\alpha}^{+}(0)+C_{\alpha}^{-} I_{\alpha}^{-}(0)\right)},
$$

where the $\alpha$ 's denote possible choices of signs for $\left(J_{b}: b \neq b^{*}\right), C_{\alpha}^{ \pm}$equals the $Z_{n}^{-1}$ appearing in (1.5) for the specified $\left(J_{b}: b \neq b^{*}\right)$ and $J_{b}= \pm\left|J_{b}\right|, H_{\alpha}=0$ or 1 according to whether the endpoints of $b^{*}$ are already connected by the $n_{b}$-bonds for $b \neq b^{*}$ or not (taking boundary conditions into account properly), and $I_{\alpha}^{ \pm}(0)$ (resp. $\left.I_{\alpha}^{ \pm}(1)\right)$ is 1 or 0 according to whether for the specified $J_{b}$ 's, the bond configuration with given $n_{b}$ for $b \neq b^{*}$ and $n_{b^{*}}=0$ (resp. $n_{b^{*}}=1$ ) belongs to $U$ (again with boundary conditions taken into account). Note that due to the independence of the boundary condition on the sign of $J_{b^{*}}, I_{\alpha}^{+}(0)=I_{\alpha}^{-}(0)$, that $I_{\alpha}^{ \pm}(0)=0$ implies $I_{\alpha}^{ \pm}(1)=0$; that $I_{\alpha}^{ \pm}(0)=1$ and $H_{\alpha}=1$ implies $I_{\alpha}^{ \pm}(1)=1$, and finally that $I_{\alpha}^{ \pm}(0)=1$ and $H_{\alpha}=0$ implies that exactly one of $\left\{I_{\alpha}^{+}(1), I_{\alpha}^{-}(1)\right\}$ is 1 . To obtain (4.4) it suffices to show that for each $\alpha$ with $I_{\alpha}^{ \pm}(0)=1$,

$$
\frac{p_{b}}{\left(1-p_{b}\right)} \frac{C_{\alpha}^{+} 2^{-H_{\alpha}} I_{\alpha}^{+}(1)+C_{\alpha}^{-} 2^{-H_{\alpha}} I_{\alpha}^{-}(1)}{C_{\alpha}^{+}+C_{\alpha}^{-}} \geq \frac{\frac{1}{2} p_{b}}{1-\frac{1}{2} p_{b}} .
$$


For $H_{\alpha}=1$, the left-hand side is $\left(p_{b} / 2\right) /\left(1-p_{b}\right)$ which obeys the inequality; for $H_{\alpha}=0$, the left-hand side is bounded below by

$$
\frac{p_{b}}{1-p_{b}} \min \left(\frac{C_{\alpha}^{+}}{C_{\alpha}^{+}+C_{\alpha}^{-}}, \frac{C_{\alpha}^{-}}{C_{\alpha}^{+}+C_{\alpha}^{-}}\right) .
$$

The desired inequality then follows from

$$
\max \left(\frac{C_{\alpha}^{+}}{C_{\alpha}^{-}}, \frac{C_{\alpha}^{-}}{C_{\alpha}^{+}}\right) \leq e^{-\beta\left|J_{b}\right|}=\frac{1}{1-p_{b}} .
$$

We leave this last inequality as an exercise for the reader (who may consult Gandolfi et al. 1992).

We suggested early in this section that FK-percolation should be a necessary condition for non-uniqueness of Gibbs distributions in spin glasses, and Theorem 3 shows that FK-percolation does occur for high $\beta$, at least for $d \geq 3$. Although there does not appear to be a proof available (it would be of interest to have such a proof), it seems that, unlike ferromagnetic models, FK-percolation should not be a sufficient condition for non-uniqueness in spin glasses. To see how this could be so, let us see how FK-percolation might be consistent with decay of the Ising spin-spin correlation, $E_{s}\left(S_{u} S_{v}\right)$, as $\|u-v\| \rightarrow \infty$.

Let us denote by $\mu_{n}$ the infinite volume FK measure under consideration (say with free or periodic b.c.); the key idea is the distinction between the general FK formula (1.8) for $E_{s}\left(S_{u} S_{v}\right)$ and the FK connectivity function, $\mu_{n}(u \leftrightarrow v)$, which is only equal to $E_{s}\left(S_{u} S_{v}\right)$ in the ferromagnetic case. The connectivity function is

$$
\mu_{n}(u \leftrightarrow v)=\mu_{n}\left(\eta_{u, v}=+1\right)+\mu_{n}\left(\eta_{u, v}=-1\right)
$$

while

$$
E_{s}\left(S_{u} S_{v}\right)=\mu_{n}\left(\eta_{v, v}=+1\right)-\mu_{n}\left(\eta_{u, v}=-1\right) .
$$

By uniqueness of the infinite FK-cluster (see Corollary 2 of Gandolfi et al. 1992), the connectivity function would not decay in the presence of percolation, but there seems to be no reason why this could not happen simultaneously with an asymptotic cancellation of the two terms of (4.10) as $\|u-v\| \rightarrow \infty$ leading to decay of $E_{s}\left(S_{u} S_{v}\right)$.

This raises the question of whether, without proving non-uniqueness of Gibbs distributions, one might at least be able to show (for appropriate $\beta$ and $d$ ) slower than exponential decay of $E_{s}\left(S_{u} S_{v}\right)$. This would demonstrate a phase transition in decay properties since one can show exponential decay for small $\beta$.

Despite the lack of results on non-uniqueness for spin glasses, let us discuss briefly some of the types of non-uniqueness which might possibly occur (for large $d$ and $\beta$ ). We base our discussion on the analysis of Newman and Stein (1992). First, it might be that there is (a.s.) non-uniqueness which is physically irrelevant because of 'weak uniqueness' (see van Enter and Fröhlich 1985 and Campanino et al. 1987). This would mean that if $\mu_{s}^{w}$ and $\mu_{s}^{\prime \omega}$ are two infinite volume Gibbs measures, obtained (like in Theorem 3) by two different $\omega$-independent boundary conditions (e.g., periodic and antiperiodic), then $\mu_{s}^{w}=\mu_{s}^{\prime w}$ for $P$-a.e. $\omega$; further $\mu_{s}^{\omega}$ would be an extremal Gibbs distribution (for $\left.\left(J_{b}(\omega)\right)\right)$ for $P$-a.e. $\omega$. A similar situation occurs 
at high temperatures in very long range spin glasses (Fröhlich and Zegarlinski 1987, Gandolfi et al. 1993).

Another possibility (see Huse and Fisher 1987 and Fisher and Huse 1987) is that (a.s.) there are exactly two extremal Gibbs distributions, related to each other by a global spin flip. In that case, for boundary conditions like free or periodic (which are unchanged by a global spin flip), $\mu_{s}^{\omega}$ would be the symmetric mixture of these two extremal distributions. A third possibility (Binder and Young 1986), based on the Parisi analysis (Parisi 1979) of the Sherrington-Kirkpatrick spin glass (Sherrington and Kirkpatrick 1975), is that, even with free or periodic b.c., many extremal Gibbs distributions would make their appearance. Presumably this would mean that $\mu_{s}^{\omega}$, obtained as discussed before Theorem 3, would have a decomposition into many extremal Gibbs distributions. On the other hand, it was argued by Newman and Stein (1992) that under this scenario, if one first fixes $\omega$ and then tries to take an infinite-volume limit, the result would be many different subsequence limits with the subsequences necessarily $\omega$-dependent. It is an open problem to effectively sort out all the various possibilities.

\section{References}

Aizenman, M., Chayes, J., Chayes, L., and Newman, C. M. (1987). The phase boundary in dilute and random Ising and Potts ferromagnets. Journal of Physics A: Mathematical and General 20, L313-L318.

Aizenman, M., Chayes, J., Chayes, L., and Newman, C. M. (1988). Discontinuity of the magnetization in one-dimensional $1 /|x-y|^{2}$ Ising and Potts models. Journal of Statistical Physics $\mathbf{5 0}$ $1-40$.

Aizenman, M., Klein, A., and Newman, C. M. (1993). Percolation methods for disordered quantum Ising models. In Phase Transitions: Mathematics, Physics, Biology, ... (R. Kotecky, ed.), World Scientific, Singapore (to appear).

Aizenman, M. and Nachtergaele, B. (1993). Geometric aspects of quantum spin systems. Communications in Mathematical Physics (to appear).

Aizenman, M. and Wehr, J. (1990). Rounding effects of quenched randomness on first-order phase transitions. Communications in Mathematical Physics 130, 489-528.

Berg, J. van den and Maes, C. (1992). Disagreement percolation in the study of Markov fields. Annals of Probability, to appear.

Binder, K. and Young, A. P. (1986). Spin glasses: Experimental facts, theoretical concepts and open questions. Review of Modern Physics 58, 801-976.

Campanino, M., Olivieri, E., and van Enter, A. C. D. (1987). One dimensional spin glasses with potential decay $1 / r^{1+\epsilon}$. Absence of phase transitions and cluster properties. Communications in Mathematical Physics 108, 241-255.

Dobrushin, R. L. (1968). The description of a random field by means of conditional probabilities and conditions of its regularity. Theory of Probability and its Applications 13, 197-224.

Dobrushin, R. L. and Shlosman, S. B. (1985). Constructive criteria for the uniqueness of a Gibbs field. In Statistical Mechanics and Dynamical Systems (J. Fritz, A. Jaffe, and D. Szász, eds.), Birkhäuser, Boston, pp. 371-403.

Edwards, S. and Anderson, P. W. (1975). Theory of spin glasses. Journal of Physics F 5, 965-974.

Enter, A. C. D. van and Fröhlich, J. (1985). Absence of symmetry breaking for $N$-vector spin glass models in two dimensions. Communications in Mathematical Physics 98, 425-432.

Edwards, R. G. and Sokal, A. D. (1988). Generalization of the Fortuin-Kasteleyn-Swendsen-Wang representation and Monte Carlo algorithm. The Physical ReviewD 38, 2009-2012.

Fisher, D. S. and Huse, D. A. (1987). Absence of many states in realistic spin glasses. Journal of Physics A: Mathematical and General 20, L1005-L1010.

Fortuin, C. M. and Kasteleyn, P. W. (1972). On the random-cluster model. I. Introduction and relation to other models. Physica 57, 536-564. 
Fröhlich, J. and Zegarlinski, B. (1987). The high-temperature phase of long-range spin glasses. Communications in Mathematical Physics 110, 121-155.

Gandolfi, A., Keane, M. S., and Newman, C. M. (1992). Uniqueness of the infinite component in a random graph with applications to percolation and spin glasses. Probability Theory and Related Fields 92, 511-527.

Gandolfi, A., Newman, C. M., and Stein, D. L. (1993). Exotic states in long range spin glasses. Communications in Mathematical Physics, to appear.

Griffiths, R. B. (1971). Phase transitions. In Statistical Mechanics and Quantum Field Theory (C. DeWitt and R. Stora, eds.), Gordon and Breach, New York, pp. 241-279.

Grimmett, G. R. (1994). Percolative problems. In Probability and Phase Transition (G. Grimmett, ed.), Kluwer, Dordrecht, pp. 69-86, this volume.

Harris, T. E. (1960). A lower bound for the critical probability in a certain percolation process. Proceedings of the Cambridge Philosophical Society 56, 13-20.

Huse, D. A. and Fisher, D. S. (1987). Pure states in spin glasses. Journal of Physics A: Mathematical and General 20, L997-L1003.

Kasai, Y. and Okiji, A. (1988). Percolation problem describing $\pm J$ Ising spin glass system. Progress in Theoretical Physics 79, 1080-1094.

Kasteleyn, P. W. and Fortuin, C. M. (1969). Phase transitions in lattice systems with random local properties. Journal of the Physical Society of Japan 26, 11-14.

Lebowitz, J. L. and Martin-Löf, A. (1972). On the uniqueness of the equilibrium state for Ising spin systems. Communications in Mathematical Physics 25, pp. 276-282.

Liggett, T. M. (1992). The survival of one-dimensional contact processes in random environments. Annals of Probability 20, 696-723.

Newman, C. M. (1991). Ising models and dependent percolation. In Topics in Statistical Dependence (H. W. Block, A. R. Sampson, and T. H. Savits, ed.), IMS Lecture Notes - Monograph Series, 16, 395-401.

Newman, C. M. and Stein, D. L. (1992). Multiple states and thermodynamic limits in short-ranged Ising spin glass models. The Physical Review B 46, 973-982.

Parisi, G. (1979). Infinite number of order parameters for spin-glasses. Physical Review Letters 43, $1754-1756$.

Reger, J. D., Bhatt, R. N., and Young, A. P. (1990). Monte Carlo study of the order-paremeter distribution in the four-dimensional Ising spin glass. Physical Review Letters 64, 1859-1862.

Sherrington, D. and Kirkpatrick, S. (1975). Solvable model of a spin glass. Physical Review Letters 35, 1792-1796.

Swendsen, R. H. and Wang, J. S. (1987). Nonuniversal critical dynamics in Monte Carlo simulations. Physical Review Letters 58, 86-88. 